\title{
A Digital Method of Noise Measurement of Charge-sensitive Preamplifiers Used with Detectors of Ionizing Radiation
}

\author{
Jin-Xian Peng, Yun Yang, Jin-Tao Zhu, Jun Yang, Xiao-Liang Luo, Guo-Fu Liu \\ College of Mechatronics and Automation \\ National University of Defense Technology \\ Changsha 410073, China \\ E-mail: jyang@nudt.edu.cn
}

\begin{abstract}
A digital method of noise measurement of chargesensitive preamplifiers (CSP) used with detectors of ionizing radiation has been proposed, which benefits from the fast development of digital techniques. Compared with the classical methods recommended by IEEE Std 301-1988, CEI/IEC 61151 and GB/T 4079-94, this digital method does not need the main amplifier and AC voltmeter. The filter and pulse shaping function of the main amplifier has been realized by a digital signal processing algorithm, which makes the digital method flexible and convenient. The design process of the digital filter and the measurement procedures have been given in detail, and the feasibility and validity of this method have been examined by a test set-up consisting of some resistors, switches, a pulse generator, a digital oscilloscope and an PC.
\end{abstract}

Keywords—noise measurement; CSP; digital method

\section{INTRODUCTION}

A preamplifier is often the first element in a signal processing chain in a particle detection system, which provides as an interface between the detector and the pulse processing and analysis electronics that follow. Its noise figure is probably the most important specification, which is normally quoted as the full-width at half-maximum (FWHM) of the response function of the detection system that is due only to the preamplifier noise and given as the equivalent energy spread in the type of detector for which the preamplifier is designed [1]. In order to improve and compare the noise performances of different preamplifiers in a wide assortment of applications, the noise level of preamplifiers should be meas

At present, there are three standards, i.e. IEEE Std 3011988 [2], CEI/IEC 61151 [3] and GB/T 4079-94 [4], being recommended to measure the noise of preamplifiers [5]. In [4], two methods, i.e. the AC (alternating-current) voltmeter method and the MCA (multi-channel analyzer) method are illustrated. Compared with the AC voltmeter method, for the implementation of the MCA method needs an appropriate detector and a multi-energy radiation source to participate, it will make the measurement at risk. Therefore, the AC voltmeter method is used more frequently and it is also the method recommended by IEEE Std 301-1988 and CEI/IEC 61151 strongly. However, the $\mathrm{AC}$ voltmeter based on the analog signal processing of the main amplifier is not only complex and costly but also vulnerable to the interference of the surroundings, which makes the noise measurement inconveniently.

More recently, with the development of digital techniques, the high performance digital electronic measurement platforms have become available with the requisite speed and cost, and therefore the digital signal processing algorithms have been implemented easily and quickly. Taking advantage of these edges, we proposed a digital implementation of measuring noise of CSP. Although the essential principle of this digital method is the same as the $\mathrm{AC}$ voltmeter method, the difference between the two methods lies in the fact that the AC voltmeter method uses analog quasi-Gaussian pulse-shaping networks of the main amplifier to filter the noise, but the digital method uses the designed quasi-Gaussian digital pulse-shaping filter to suppress the noise. Based on the inherent virtues of digital techniques, the digital method has the advantages of computation economy, performance stability and antiinterference capacity, which makes it suitable for noise measurement applications.

In this paper, a brief introduction of the $\mathrm{AC}$ voltmeter method is given in Section 2; the measurement principle and detailed measurement procedures of the digital method are given in Section 3; the experimental results and discussions are given in Section 4; the conclusions arising from this research are given in section 5 .

\section{AC VOLTMETER METHOD}

The essential principle of noise measurement of the AC voltmeter method is introduced as follows: first of all, obtaining the RMS (root-mean-square) value of the noise of CSP filtered by the quasi-Gaussian pulse-shaping filter; and then correcting for all used instruments noise except the CSP; finally, the result must be equivalent to the input of the CSP. According to this measurement principle, the following test procedures and noise correction method are given.

\section{A. Test procedures for noise}

First, confirm that you have the correct template for your paper size. This template has been tailored for output on the US-letter paper size. If you are using A4-sized paper, please close this file and download the file "MSW_A4_format". 


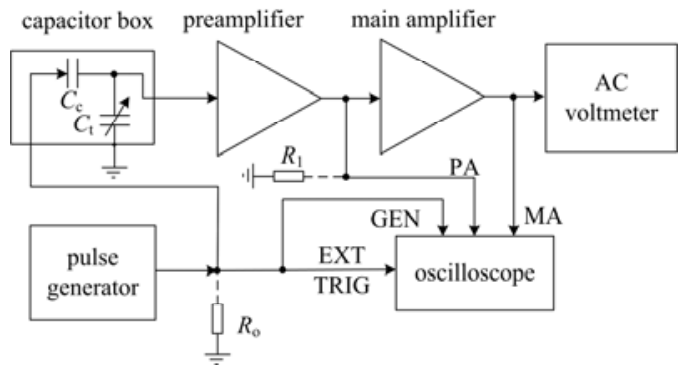

Fig. 1. The test set-up for the AC voltmeter method

The test set-up for the AC voltmeter method is shown in Fig.1. Before the measurement, the baseline restorer masking effect, if present, shall be eliminated, and a search for correlated noise shall be made. The pole/zero compensation shall be adjusted if necessary. The specific steps are as follows:

$A$. Set the (simulated) detector capacitance $C_{\mathrm{t}}$ to one end of the desired range and adjust the generator and main amplifier to meet the conditions: the input pulse amplitude of the preamplifier $V_{\mathrm{p}}$ and the output pulse amplitude of the main amplifier $V_{\mathrm{o}}$ cannot be too small.

$B$. Turn off the generator. If it is disconnected, a terminating resistor $R_{\mathrm{o}}$ must be left in place at the input of the capacitor box to prevent external noise pickup, and record the indication eno of the $\mathrm{AC}$ voltmeter.

Substituting the above parameters into Eq. (1), we can obtain the equivalent input noise charge (ENC) in FWHM of the preamplifier when $C_{\mathrm{t}}$ is one end of the desired range:

$$
\mathrm{FWHM}_{\mathrm{NC}}=1.128 \times 2.355 \frac{V_{\mathrm{p}} C_{\mathrm{c}}}{V_{\mathrm{o}}} e_{\mathrm{no}}
$$

where 1.128 is the scale factor multiplied by the meter reading to obtain the RMS voltage with a Gaussian noise and 2.355 is the conversion factor multiplied by the RMS voltage to obtain the FWHM value.

When changing $C_{\mathrm{t}}$ for a sequence of noise measurements, the output pulse amplitude shall be kept fixed throughout the run by readjustment of the main amplifier gain at each change in capacitance. The shaping index and the generator setting shall also be fixed throughout the sequence.

\section{B. Noise correction}

The measured preamplifier noise shall be corrected for the main amplifier noise in the following way: after determining eno, disconnect the preamplifier and terminate the input of the main amplifier with an impedance R1 which is the same value as the output impedance of the preamplifier. Record the new enos. The corrected noise due to the preamplifier alone is the quadratic difference $\left[\left(e_{\mathrm{no}}\right)^{2}-\left(e_{\mathrm{nos}}\right)^{2}\right]^{1 / 2}$. Therefore, the noise after corrected formula is

$$
\mathrm{FWHM}_{\mathrm{NC}}=1.128 \times 2.355 \frac{V_{\mathrm{p}} C_{\mathrm{c}}}{V_{\mathrm{o}}} \sqrt{e_{\mathrm{no}}^{2}-e_{\text {nos }}^{2}}
$$

\section{Digital MeTHoD}

The AC voltmeter method analyzed above is based on the analog signal processing, which is time-consuming and inconvenient. With the development of high-speed signal acquisition techniques, it becomes increasingly possible to sample the signals from the preamplifier directly. Consequently, a new method based on digital signal processing was developed in this paper. Compared with the AC voltmeter method, the digital method is simple, efficient, and low-cost [5-7]. Furthermore, since the signal is digitized early, it becomes more stable and reliable during the transmission and processing.

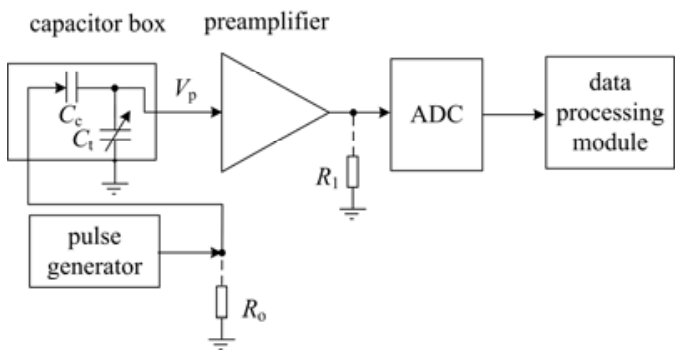

Fig. 2. The test set-up for the digital method

The test set-up for the digital method is shown in Fig.2. The signal pulse from the preamplifier is directly digitized by a fast ADC, and then shaped into a quasi-Gaussian pulse by a digital filter designed and stored in the data processing module. Digital filters, unlike their analogue counterparts, do not have decaying tails, and digital pulse-shaping filters have a greater potential in terms of the throughput without the degradation of energy resolution [8]. Therefore, the quasiGaussian shaping of digital filter can be used to measure the noise of CSP instead of the main amplifier.

According to the above measurement principle of the digital method, the specific operation procedures and detailed deduction of the formula are given as follows:

A. Set the (simulated) detector capacitance $C_{t}$ to one end of the desired range. Turn off the generator, and sample the output noise from the preamplifier by an acquisition system, and then record the RMS of the noise filtered by the designed digital filter $e_{n o}$.

B. In order to correct the acquisition system noise, operate in the following way: after determining eno, disconnect the preamplifier and terminate the input of the acquisition system with an impedance R1 which is the same value as the output impedance of the preamplifier, and record the new enos. The corrected noise expressed in FWHM is 


$$
\mathrm{FWHM}_{\mathrm{NC}}=1.128 \times 2.355 \frac{V_{\mathrm{p}} C_{\mathrm{c}}}{V_{\mathrm{o}}} e_{\mathrm{no}}
$$

C. To obtain the noise in units of equivalent input charge in FWHM, adjust the output amplitude $V_{p}$ from pulse generator, the signal is sampled by the acquisition system and shaped by the designed digital filter, and then the magnitude of the digital filter output is $V_{o}$. Since the input charge is $V_{p} C_{c}$, thus

$$
\frac{\text { FWHM }}{\text { FWHM }_{\mathrm{NC}}}=\frac{V_{\mathrm{o}}}{V_{\mathrm{p}} C_{\mathrm{c}}}
$$

Substituting Eq. (4) into Eq. (3), it follows that

$$
\mathrm{FWHM}_{\mathrm{NC}}=2.355 \frac{V_{\mathrm{p}}}{V_{\mathrm{o}}} C_{\mathrm{c}} \sqrt{e_{\mathrm{no}}^{2}-e_{\mathrm{nos}}^{2}}
$$

where units are in farad and volt.

D. Change $C_{t}$ and repeat, we can get the relationship between the noise level and $C_{t}$.

The noise of the CSP can also be expressed as equivalent input noise energy (ENE), equivalent input noise voltage (ENV), and equivalent input noise ion pairs [3]:

$$
\begin{gathered}
\mathrm{FWHM}_{\mathrm{NE}}=2.355 \frac{\varepsilon V_{\mathrm{p}}}{q V_{\mathrm{o}}} C_{\mathrm{c}} \sqrt{e_{\mathrm{no}}^{2}-e_{\mathrm{nos}}^{2}} \\
\text { FWHM }_{\mathrm{NV}}=2.355 \frac{V_{\mathrm{p}}}{V_{\mathrm{o}}} \sqrt{e_{\mathrm{no}}^{2}-e_{\mathrm{nos}}^{2}} \\
\mathrm{FWHM}_{\mathrm{NI}}=2.355 \frac{V_{\mathrm{p}}}{q V_{\mathrm{o}}} C_{\mathrm{c}} \sqrt{e_{\mathrm{no}}^{2}-e_{\mathrm{nos}}^{2}}
\end{gathered}
$$

where $q$ is the electronic charge and $\varepsilon$ is the average ionization energy of the detector.

For different detectors including preamplifiers used together, the units of noise are different. However, a suitable unit is always recommended, such as gridded ionization chambers, germanium, and silicon detectors, the noise shall be specified in $\mathrm{eV}$ or $\mathrm{keV}$ FWHM for the applicable detector.

\section{EXPERIMENTAL RESULTS AND DISCUSSIONS}

We will analyze the performance of the AC voltmeter method and the digital method by measuring the inherent noise of the preamplifier (i.e. $C_{\mathrm{t}}=0$ ) manufactured by our laboratory.

The noise measurement set-ups for the AC voltmeter method and the digital method have been shown in Fig. 1 and Fig. 2 respectively. In both set-ups, the pulse generator is the
Agilent 33522A with 30MHz Function/Arbitrary Waveform Generator. A digital oscilloscope with a sampling rate of $5 \mathrm{G}$ Sample/s and 8-bit resolution DPO4104 is used as an acquisition system to capture the signal pulse from the preamplifier, and the filter is realized in MATLAB environment.

\section{A. Design of digital filter}

As we all know, the art of analog filter design is highly advanced, and since useful results can be achieved, it is advantageous to use the design procedures already developed for analog filters. Impulse invariance method and bilinear transformation method are often used to design a digital filter by transforming a prototype analog filter.

In the $\mathrm{AC}$ voltmeter method, the transfer function of the main amplifier used in Fig. 1 is

$$
H_{\mathrm{a}}(s)=\frac{4\left(s+1 / \tau_{1}\right) / \tau^{4}}{(s+1 / \tau)\left(s^{2}+2 s / \tau-2 / \tau^{2}\right)^{2}}
$$

where $\tau_{1}$ is the decay time constant of the output from the preamplifier and $\tau$ is the time constant of pulse-shaping network of the main amplifier.

By using the bilinear transformation method, we can obtain the Z-transfer function of this analog filter with a sampling frequency $f_{\mathrm{s}}$

$$
H_{\mathrm{d}}(z)=\frac{b_{0}+b_{1} z^{-1}+b_{2} z^{-2}+\cdots+b_{5} z^{-5}}{a_{0}+a_{1} z^{-1}+a_{2} z^{-2}+\cdots+a_{5} z^{-5}}
$$

\begin{tabular}{|c|c|c|c|c|c|c|}
\hline \multirow{2}{*}{$\begin{array}{c}\text { coefficie } \\
\text { nts }\end{array}$} & \multicolumn{6}{|c|}{ i } \\
\hline & 0 & 1 & 2 & 3 & 4 & 5 \\
\hline $\begin{array}{c}a_{\mathrm{i}} \\
\left(\times 10^{12}\right)\end{array}$ & 1.0257 & $\begin{array}{c}- \\
4.6222\end{array}$ & 8.3489 & $\begin{array}{c}- \\
7.5553\end{array}$ & 3.4254 & $\begin{array}{c}- \\
0.6224\end{array}$ \\
\hline $\begin{array}{c}b_{\mathrm{i}} \\
\left(\times 10^{7}\right)\end{array}$ & 2.0050 & 6.0250 & 4.0500 & $\begin{array}{c}- \\
3.9500\end{array}$ & $\begin{array}{c}- \\
5.9750\end{array}$ & $\begin{array}{c}- \\
1.9950\end{array}$ \\
\hline
\end{tabular}

where the coefficients $a_{\mathrm{i}}$ and $b_{\mathrm{i}}(\mathrm{i}=0,1, \ldots 5)$ can be calculated according to the values of $\tau_{1}, \tau$ and $f_{\mathrm{s}}$. In the experimental, $\tau_{1}=20 \mu \mathrm{s}, \tau=1 \mu \mathrm{s}$ and $f_{\mathrm{s}}=10^{7} \mathrm{~Hz}$, the values of $a_{\mathrm{i}}$ and $b_{\mathrm{i}}$ are shown in Table I.

TABLE I. The VAlues of CoefFicients Ai AND BI

Having had the Z-transfer function of the filter, the recursive form (difference equation) of the filter is obtained as

$$
y[n]=\sum_{i=1}^{5} a_{\mathrm{i}} y[n-i]+\sum_{i=0}^{5} b_{\mathrm{i}} x[n-i]
$$

where $x[n]$ is the sampled preamplifier data, $y[n]$ is the filtered signal and $n$ is the sample index. 


\section{B. Output of the digital filter}

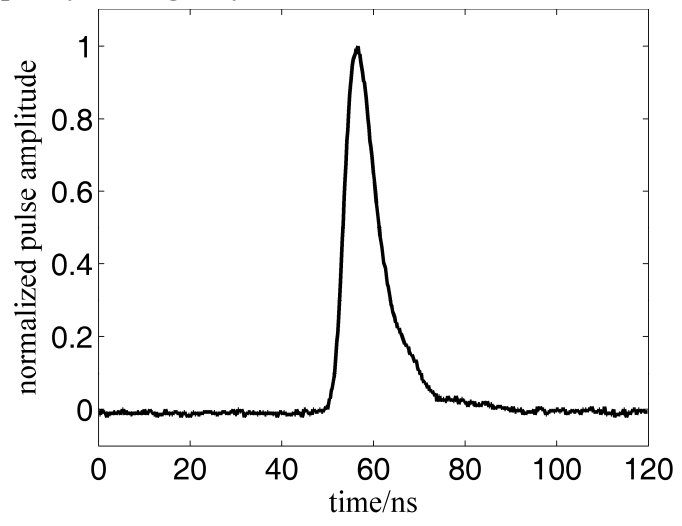

Fig. 3. The output signal from PMT

The output from the photomultiplier tube (PMT) of the liquid scintillation detector filled with $\mathrm{BC} 501 \mathrm{~A}$ organic liquid is shown in Fig.3. The integrated signal by the CSP, the response of the digital filter and the output of the main amplifier are shown in Fig.4.

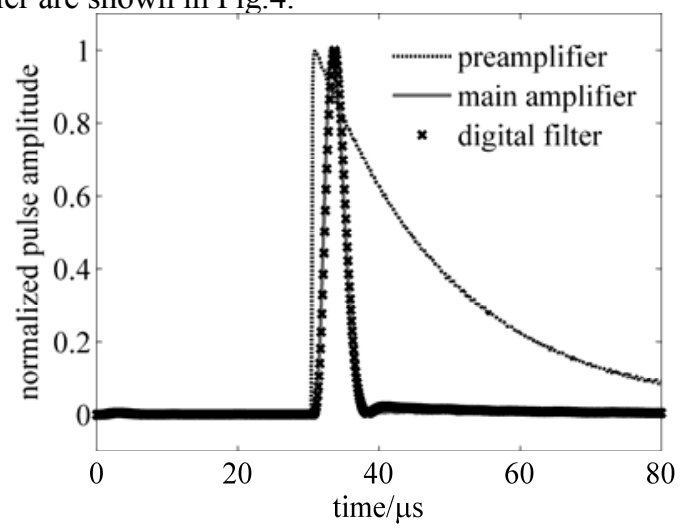

Fig. 4. The outputs from the preamplifier, the main amplifier and the digital filter

From Fig.4, it can be seen that the output of the digital filter is consistent with that of the main amplifier.

\section{Experimental results}

1) Results of the $A C$ voltmeter method. In Fig.1, the function of $\mathrm{AC}$ voltmeter is completed by the digital oscilloscope in the experiment. The signal pulse from the main amplifier is captured, and then the magnitude of signal is analyzed and RMS of the noise is calculated. Therefore, the measured preamplifier noise must be corrected for the main amplifier and the acquisition system noises. The relevant parameters are presented in Table II.

TABLE II. PARAMETERS FOR THE AC VOLTMETER METHOD

\begin{tabular}{|c|c|c|c|c|}
\hline$C_{\mathrm{c}} / \mathrm{pF}$ & $V_{\mathrm{p}} / \mathrm{V}$ & $V_{\mathrm{o}} / \mathrm{V}$ & $\begin{array}{c}e_{\text {no }} / \mathrm{V} \\
\left(\times 10^{-4}\right)\end{array}$ & $\begin{array}{c}e_{\text {nos }} / \mathrm{V} \\
\left(\times 10^{-4}\right)\end{array}$ \\
\hline 200 & 1.5 & 0.2751 & 2.3856 & 2.2842 \\
\hline
\end{tabular}

Substituting the parameters in Table 2 into Eq. (2), we can obtain the result

$$
\begin{aligned}
& \mathrm{FWHM}_{\mathrm{NC}}=2.355 \frac{V_{\mathrm{p}} C_{\mathrm{c}}}{V_{\mathrm{o}}} \sqrt{e_{\mathrm{no}}^{2}-e_{\mathrm{nos}}^{2}} \\
& =1.7672 \times 10^{-13} \mathrm{C}
\end{aligned}
$$

In this condition, since we had obtained the true RMS value of noise, the conversion factor 1.128 is unnecessary.

2) Results of the $A C$ voltmeter method. Before the experiment, the digital filter has been designed and stored in the computer. According to the specific operation procedures in Section 3, the relevant parameters of digital method are presented in Table III.

TABLE III. PARAMETERS FOR THE DIGITAL METHOD

\begin{tabular}{|c|c|c|c|c|}
\hline $\boldsymbol{C}_{\mathrm{c}} / \mathbf{p F}$ & $\boldsymbol{V}_{\mathrm{p}} / \mathbf{V}$ & $\boldsymbol{V}_{\mathbf{o}} / \mathbf{V}$ & $\begin{array}{c}\boldsymbol{e}_{\mathbf{n o}} / \mathbf{V} \\
\left(\times \mathbf{1 0}^{-4}\right)\end{array}$ & $\begin{array}{c}\boldsymbol{e}_{\mathbf{n o s}} / \mathbf{V} \\
\left(\times \mathbf{1 0}^{-4}\right)\end{array}$ \\
\hline 200 & 1.5 & 0.4703 & 1.0669 & 3.0019 \\
\hline
\end{tabular}

Substituting the parameters in Table 3 into Eq. (5), we can obtain

$$
\begin{aligned}
& \mathrm{FWHM}_{\mathrm{NC}}=2.355 \frac{V_{\mathrm{p}}}{V_{\mathrm{o}}} C_{\mathrm{c}} \sqrt{e_{\mathrm{no}}^{2}-e_{\mathrm{nos}}^{2}} \\
& =1.5380 \times 10^{-13} \mathrm{C}
\end{aligned}
$$

As the condition in the AC voltmeter method, the conversion factor 1.128 is unnecessary.

\section{Discussion}

Compared with the AC voltmeter method, the digital method can lead to a better noise measurement result. The reason is that the impact of the main amplifier cannot be corrected completely for the AC voltmeter method, and this is the fatal drawback of the classical methods. What's more, the interference of the surrounding also has an impact on the results. On the contrary, the digital method does not have these problems, and it suppresses the noise from the preamplifier effectively.

\section{CONCLUSION}

We have presented a noise measurement method based on digital signal processing for CSP used with detectors of ionizing radiation. The digital method can be realized without main amplifier, and it has been proved to be a very simple, efficient, and low-cost method for noise measurement for CSP. With the development of digital techniques, this method will have a good prospect in the noise measurement of CSP.

\section{ACKNOWLEDGEMENT}

Supported by the National Natural Science Foundation of China (11175254)ured. 


\section{REFERENCES}

[1] Glenn F. Knoll. Radiation Detection and Measurement (the fourth edition). Wiley Press, 2010.

[2] IEEE Standard Test Procedures for Amplifiers and Preamplifiers used with Detectors of Ionizing Radiation, IEEE Std. 301-1988, 1988.

[3] Nuclear instrumentation-Amplifiers and preamplifiers used with detectors of ionizing radiation- Test procedures, CEI/IEC 61151, 1992.
[4] Test procedures for amplifiers and charge-sensitive preamplifiers used with detectors of ionizing radiation, GB/T 4079-94, 1994.

[5] M. Nakhostin. Nucl. Nucl. Instrum. Methods A, 2012, 672: 1-5

[6] M. Nakhostin. IEEE Trans. Nucl. Sci. 2011, 58: 2378

[7] M. Nakhostin, P.M. Walker. Nucl. Nucl. Instrum. Methods A, 2010, 621: 498-501

[8] Vladimir Polushkin. Nuclear Electronics Superconducting Detectors and Processing Techniques. England: John Wiley \& Sons Ltd. 2004, 193-198 